\title{
High-Quality Genome Resource of Diaporthe destruens Causing Foot Rot Disease of Sweet Potato
}

\author{
Lifei Huang, ${ }^{1}$ Xinxin Zhang, ${ }^{1}$ Yiling Yang, ${ }^{1}$ Hongda Zou, ${ }^{1}$ Boping Fang, ${ }^{1, \dagger}$ and Weiming Liu ${ }^{2, \dagger}$ \\ ${ }^{1}$ Crop Research Institute, Guangdong Academy of Agricultural Sciences, Guangdong Provincial Key \\ Laboratory of Crops Genetics and Improvement, Guangzhou, Guangdong, China \\ ${ }^{2}$ Taizhou Vocational College of Science \& Technology, Taizhou, Zhejiang, China
}

\begin{abstract}
Foot rot of sweet potato caused by Diaporthe destruens severely affects yield and quality worldwide. Research on this pathogen is limited due to nonavailability of genome resources. Here, we report a high-quality genome sequence of $D$. destruens isolate CRI 305-2, which was originally isolated from infected stem of sweet potato in Taizhou City, Zhejiang Province, China. The genome comprised a total length of 56,108,228 bp, consisted of 47 scaffolds with an overall $\mathrm{G}+\mathrm{C}$ content of $48.7 \%$ and an $\mathrm{N} 50$ of $2,479,481 \mathrm{bp}$. This resource can be used as a reference for evolution mechanisms and comparative genomic research.
\end{abstract}

Diaporthe destruens (Harter) Hirooka, Minosh. \& Rossman is a pathogenic fungus that causes foot rot of sweet potato (synonyms: Phomopsis destruens (Harter) Boerema Loer. \& Hamers, Plenodomus destruens Harter). This fungus causes the disease on seedlings, mature plants, and storage roots. It was first discovered in the United States in 1912 with an incidence rate of $95 \%$ and yield loss rate of 10 to $50 \%$ (Harter 1913). In recent years, the disease has caused significant threats to sweet potato production in China, Korea, Japan, Brazil, Argentina, and Uruguay (Clark et al. 2013; Gai et al. 2016; Paul et al. 2019). Sweet potato foot rot has reached epidemic proportions in Zhejiang Province, China, with an average incidence rate of 10 to $20 \%$; incidence rates as high as $100 \%$ were recorded in some fields that resulted in considerable economic losses (Huang et al. 2019). In 2015, D. destruens isolate CRI 305-2 was isolated from infected sweet potato stems in Taizhou City, Zhejiang Province, China. The fungus was identified as $D$. destruens based on morphological traits, and a phylogenetic tree was obtained from the alignment of internal transcribed spacer (ITS) and translation elongation factor 1- $\alpha(E F-1 \alpha)$ genes. There are currently no available genome resources for research on this pathogen. Here, we report a high-quality genome sequence of $D$. destruens isolate CRI 305-2, which was originally isolated from infected stem of sweet potato in Taizhou City, Zhejiang Province, China.

D. destruens isolate $\mathrm{CRI} 305-2$ was cultured in liquid nutrient agar for 7 days at $28^{\circ} \mathrm{C}(\mathrm{pH}$ 6.0) and $120 \mathrm{rpm}$. Genomic DNA was extracted using Qiagen genomic tip-100/G DNA isolation kit (QIAGEN Inc., Hilden, Germany) according to the manufacturer's protocol. DNA quality and concentration were assessed using $0.5 \%$ agarose gel electrophoresis and Qubit 3.0 fluorimeter (Invitrogen, Carlsbad, CA, U.S.A.). The DNA size ranged from 20 to $150 \mathrm{~kb}$, an A260/A280 ratio of 1.89 , and concentration of $230.0 \mathrm{ng} / \mu \mathrm{l}$ were recorded. Whole-genome sequencing data were obtained using the Oxford Nanopore Technology (ONT) PromethION platform (Oxford Nanopore, Oxford, UK) and short-read Illumina on an Illumina HiSeq 4000 sequencer.

A total of 812,526 raw reads $(6.75 \mathrm{~Gb})$ with $\mathrm{N} 50$ of $15,608 \mathrm{bp}$ generated were filtered to remove short reads ( $<2 \mathrm{~kb})$, low-quality bases, and adapter sequences. For Illumina-based

${ }^{\dagger}$ Corresponding authors: B. P. Fang; fangboping@gdaas.cn, and W. M. Liu; Iwm4567878@163.com

The author(s) declare no conflict of interest.

Accepted for publication 21 March 2021.
Funding

This research was supported by the Key-Area Research and Development Program of Guangdong Province (no. 2020B020219001), National Sweet Potato Industry Technical System (no. CARS-10-B-05 and CARS-10-C-17), and Sweet Potato Potato Innovation Team of Modern Agricultural Industry Technology System in Guangdong Province (2019KJ111).

\section{Keywords}

sweet potato, foot rot, genome, pathogen, Diaporthe destruens 
Table 1. Genome statistics of Diaporthe destruens isolate 305-2

\begin{tabular}{lc} 
Feature & D. destruens \\
Depth of Nanopore sequence & $121 \times$ \\
Depth of Illumina sequence & $79 \times$ \\
Assembly size (bp) & $56,108,228$ \\
Number of scaffolds & 47 \\
N50 length (bp) & $2,479,481$ \\
N90 length (bp) & 878,686 \\
Scaffold max (bp) & $6,293,594$ \\
GC content (\%) & 48.7 \\
BUSCO completeness (\%) & 97.93 \\
Repeat rate (\%) & 3.33 \\
Annotation & \\
Predicted protein-coding gene & 13,754 \\
Annotated nonredundant protein sequences & 12,645 \\
Secreted proteins & 1,197 \\
Secreted carbohydrate active enzyme & 917 \\
Pathogen-host interaction (PHI) proteins & 4,158 \\
GenBank accession no. & JACAAM000000000 \\
\hline
\end{tabular}

polishing, a total of $16,639,343$ raw reads of $2 \times 150$ paired end $(2.50 \mathrm{~Gb})$ were yielded by the Illumina sequencing, out of which $91.62 \%$ had a Phred Q score $>30$. Adapters and low-quality bases were removed from Illumina reads using Trimmomatic version 0.38 (Bolger et al. 2014) with conservative parameters to remove reads with a quality score $<Q 3$ and short length $<25$ bp. A total of 623,766 high-quality reads $(6,836,107,246$ bp; $6.37 \mathrm{~Gb})$ with 121 -fold coverage were obtained, which were assembled using wtdbg2 version 2.3 with parameters: $-\mathrm{H}-\mathrm{k} 21$ -j 1000 -S 1.02 -e 3 (Ruan and Li 2020) and Pilon version 1.23 (Walker et al. 2014) with default parameters, which was run for five rounds. The genome assembly was $56,108,228 \mathrm{bp}$ in length, consisted of 47 scaffolds with an overall $\mathrm{G}+\mathrm{C}$ content of $48.7 \%$, an N50 of 2,479,481 bp, and an N90 of $878,686 \mathrm{bp}$; the longest scaffold contained 6,293,594 bp (Table 1).

The combination of short Illumina read data and long Nanopore read data has been used to reduce errors and improve the quality of reference genomes. The paired-end library with an average insert size of 350 bp was constructed following the standard Illumina Library construction and sequencing protocol (San Diego, CA, U.S.A.). Illumina reads with 79-fold coverage were aligned to the $D$. destruens genome assembly using Burrows-Wheeler alignment tool (Li and Durbin 2009). Approximately $98.29 \%$ of the clean reads mapped to the genome assembly. This indicates an appropriate assembled genome from the Nanopore data.

The completeness of the assembly was verified with BUSCO v2.0 software (Simão et al. 2015), which is based on a set of 290 common fungal genes. About $97.93 \%$ (284/290) of the BUSCOs in full length genes were found in the assembly. We constructed a de novo repeat library using RepeatModeler2 (Flynn et al. 2020), which employing two modules of RepeatScout (Price et al. 2005) and RECON (Bao and Eddy 2002), with default parameters. The library was searched as the reference database, and repeats were predicted using RepeatMasker version 4.1.0 (Smit et al. 2013). A total of 1,870,522 bp (1.87 Mb) of repeat sequences that accounted for $3.33 \%$ of the assembly, including DNA transposon, LTR retrotransposon, Tandem Repeat sequence and other transposon, were detected in the genome assembly.

A total of 13,754 protein-coding genes were predicted using ab initio-based, homologbased, and RNA-sequencing (RNA-seq)-based methods by EVM (version 1.1.1) and PASA (version 2.0.2) (Campbell et al. 2006; Haas et al. 2008). Approximately $97.93 \%(12,645 /$ 13,754 ) of the predicted genes were aligned in the nonredundant protein sequences (NR) using NCBI BLASTP (version 2.2.31) with e-value 5 and parameter -max_target_seqs 1. A total of 4,158 genes involved in pathogen-host interactions $(\mathrm{PHI})$ were predicted using $\mathrm{PHI}$ database v4.8 (Urban et al. 2017); 917 carbohydrate enzyme (CAZYmes) genes were annotated based on the dbCAN-seq database (Huang et al. 2018) using HMMER software (Eddy 1998). Additionally, 1,598 secreted proteins were predicted by SignalP 4.0 (Petersen et al. 2011).

This study reports a high-quality $D$. destruens draft genome assembly, which will serve as a reference genome for molecular genetic marker development, gene cloning and analysis, propagation and evolution mechanisms, and comparative genomics. This Whole Genome 
Shotgun project has been deposited at DDBJ/ENA/GenBank under the accession JACAAM000000000. The version described in this paper is version JACAAM010000000.

The $D$. destruens isolate used in this study is available at the Agricultural Culture Collection of China (ACCC) in Beijing and at the Crops Research Institute Guangdong Academy of Agricultural Sciences in Guangzhou, China.

\section{Literature Cited}

Bao, Z., and Eddy, S. R. 2002. Automated de novo identification of repeat sequence families in sequenced genomes. Genome Res. 12:1269-1276.

Bolger, A. M., Lohse, M., and Usadel, B. 2014. Trimmomatic: A flexible trimmer for Illumina Sequence Data. Bioinformatics 30:2114-2120.

Campbell, M. A., Haas, B. J., Hamilton, J. P., Mount, S. M., and Buell, C. R. 2006. Comprehensive analysis of alternative splicing in rice and comparative analyses with Arabidopsis. BMC Genomics 7:327.

Clark, C. A., Ferrin, D. M., Smith, T. P., and Holmes, G. J., eds. 2013. Compendium of sweetpotato diseases, pests, and disorders. APS Press, St. Paul, MN.

Eddy, S. R. 1998. Profile hidden Markov models. Bioinformatics 14:755-763.

Flynn, J. M., Hubley, R., Goubert, C., Rosen, J., Clark, A. G., Feschotte, C., and Smit, A. F. 2020. RepeatModeler2 for automated genomic discovery of transposable element families. Proc. Natl. Acad. Sci. USA 117:9451-9457.

Gai, Y. P., Ma, H. J., Chen, X. L., Zheng, J. Y., Chen, H. H., and Li, H. Y. 2016. Stem blight foot rot and storage tuber rot of sweetpotato caused by Plenodomus destruens in China. J. Gen. Plant Pathol. 82:181-185.

Haas, B. J., Salzberg, S. L., Zhu, W., Pertea, M., Allen, J. E., Orvis, J., White, O., Buell, C. R., and Wortman, J. R. 2008. Automated eukaryotic gene structure annotation using evidence modeler and the program to assemble spliced alignments. Genome Biol. 9:R7.

Harter, L. 1913. Foot-rot, a new disease of the sweet potato. Phytopathology 3:243-245.

Huang, L., Zhang, H., Wu, P., Entwistle, S., Li, X., Yohe, T., Yi, H., Yang, Z., and Yin, Y. 2018. dbCAN-seq: a database of carbohydrate-active enzyme (CAZyme) sequence and annotation. Nucleic Acids Res. 46 (D1):D516-D521.
Huang, L. F., Liu, W. M., Liu, Y. N., and He, X. B. 2019. Investigation on the stem base rot of sweetpotato and identification of pathogen species. Chin. Agric. Sci. Bull. 35:135141.

Li, H., and Durbin, R. 2009. Fast and accurate short read alignment with Burrows-Wheeler Transform. Bioinformatics 25:1754-1760

Paul, N. C., Nam, S.-S., Park, W., Yang, J. W., and Kachroo, A. 2019. First report of storage tuber rot in sweetpotato (Ipomoea batatas) caused by Plenodomus destruens in Korea. Plant Dis. 103:1020.

Petersen, T. N., Brunak, S., von Heijne, G., and Nielsen, H. 2011. SignalP 4.0: discriminating signal peptides from transmembrane regions. Nat. Methods 8:785-786.

Price, A. L., Jones, N. C., and Pevzner, P. A. 2005. De novo identification of repeat families in large genomes. Bioinformatics 21 (Suppl 1):i351-i358.

Ruan, J., and Li, H. 2020. Fast and accurate long-read assembly with wtdbg2. Nat. Methods 17:155-158.

Simão, F. A., Waterhouse, R. M., loannidis, P., Kriventseva, E., and Zdobnov, E. M. 2015. BUSCO: assessing genome assembly and annotation completeness with single-copy orthologs. Bioinformatics 31:3210-3212.

Smit, A. F. A., Hubley, R., Green, P. 2013. RepeatMasker Open-4.0. https://www. repeatmasker.org/

Urban, M., Cuzick, A., Rutherford, K., Irvine, A., Pedro, H., Pant, R., Sadanadan, V., Khamari, L., Billal, S., Mohanty, S., and Hammond-Kosack, K. E. 2017. PHlbase: A new interface and further additions for the multi-species pathogen-host interactions database. Nucleic Acids Res. 45:D604-D610.

Walker, B. J., Abeel, T., Shea, T., Priest, M., and Earl, A. M. 2014. Pilon: an integrated tool for comprehensive microbial variant detection and genome assembly improvement PLoS One 9:e112963. 